\title{
Common Fixed Point Theorem in Semi-metric Space with Compatible mapping of Type (E)
}

\author{
${ }^{1 *} \mathrm{U}$. Rajopadhyaya, ${ }^{1} \mathrm{~K}$. Jha, and ${ }^{2} \mathrm{M}$. Imdad \\ ${ }^{1}$ Department of Natural Sciences (Mathematics), \\ School of Science, Kathmandu University, Nepal \\ ${ }^{2}$ Department of Mathematics, Aligarh Muslim University, Aligarh, India. \\ ${ }^{*}$ Corresponding Author
}

Keywords: Semi-metric space, compatible mapping of type (E), common fixed point.

\begin{abstract}
In this paper, we establish a common fixed point theorem in semi-metric space with compatible mapping of type (E) which improves and extends similar known results in the literature.
\end{abstract}

\section{Introduction}

Banach contraction principle is a well known fundamental result in fixed point theory which has been used and extended in many different directions. There have been several generalizations of metric space. Among which one of the generalization is semi-metric space initiated by M.Frechet [5], K. Menger [12] and W.A. Wilson[15]. M. Cicchese[4] introduced the notion of contractive mapping in semi-metric space and proved the first fixed point theorem for this class of space. M. Aamri and D.El. Moutawakil[1] gave a notion of E.A. property which generalize the concept of non-compatible mappings in metric space and proved some common fixed point theorems for noncompatible mappings under strict contractive conditions. G.Jungck[11] introduced the concept of weakly compatible mapping. This concept has been frequently used to prove existence theorem in common fixed point theory.

Among various type of compatible mapping in 2007, M.R. Singh and Y. M. Singh[14] introduced the concept of compatible mapping of type (E). In this paper we prove a common fixed point theorem for two pairs of self mappings using compatible mapping of type (E) in semimetric space by extending the results of M. Aamri and D.El. Moutawakil[1] and other similar results.

Let $X$ be a non-empty set and $d: X \times X \rightarrow[0, \infty) .(X, d)$ be a semi-metric space (symmetric space) if and only if it satisfies the following:

W1 $: d(x, y)=0$ if and only if $x=y$.

W2: $d(x, y)=d(y, x)$ if and only if $x=y$ for any $\quad x, y \in X$

The difference of a semi-metric and a metric comes from the triangle inequality. In order to obtain fixed point theorems on a semi-metric space, we need some additional axioms which are as follows.

Let $(X, d)$ be a semi-metric space. Then, for sequence $\left\{x_{n}\right\},\left\{y_{n}\right\}$ in $X$ and $x \in X$, we have

W4[15]: $\lim _{n \rightarrow \infty} d\left(x_{n}, x\right)=0$ and $\lim _{n \rightarrow \infty} d\left(y_{n}, x_{n}\right)=0$ imply $\lim _{n \rightarrow \infty} d\left(y_{n}, x\right)=0$.

H.E[1].: $\lim _{n \rightarrow \infty} d\left(x_{n}, x\right)=0 \lim _{n \rightarrow \infty} d\left(y_{n}, x\right)=0$ imply $\lim _{n \rightarrow \infty} d\left(y_{n}, x_{n}\right)=0$.

Definition 1.1 [1] Let $A$ and $B$ be two self-mappings of a semi-metric space $(X, d)$. Then $A$ and $B$ are said to be compatible if $\lim _{n \rightarrow \infty} d\left(A B x_{n}, B A x_{n}\right)=0$, whenever $\left\{x_{n}\right\}$ is a sequence in $X$ such that $\lim _{n \rightarrow \infty} d\left(A x_{n}, t\right)=\lim _{n \rightarrow \infty} d\left(B x_{n}, t\right)=0$, for some $t \in X$. 
Definition 1.2. [1] Let $A$ and $B$ be two self-mappings of a semi-metric space $(X, d)$. Then $A$ and $B$ satisfy the property $E . A$ if there exists a sequence $\left\{x_{n}\right\}$ such that $\lim _{n \rightarrow \infty} d\left(A x_{n}, t\right)=\lim _{n \rightarrow \infty} d\left(B x_{n}, t\right)=0$, for some $t \in X$.

Definition 1.3. [14] Let $A$ and $B$ be two self-mappings of a semi-metric space $(X, d)$. Then $A$ and $B$ are said to be compatible mapping of type (E)

if $\lim _{n \rightarrow \infty} A A x_{n}=\lim _{n \rightarrow \infty} A B x_{n}=B(t) \lim _{\text {and } n \rightarrow \infty} B B x_{n}=\lim _{n \rightarrow \infty} B A x_{n}=A(t)$, whenever $\left\{x_{n}\right\}$ is a sequence in $\mathrm{X}$ such that $\lim _{n \rightarrow \infty} d\left(A x_{n}, t\right)=\lim _{n \rightarrow \infty} d\left(B x_{n}, t\right)=0$, for some $t \in X$.

\section{Proposition 1.1[10]:}

Let $\mathrm{A}$ and $\mathrm{B}$ be two compatible mappings of type (E). If one of the function is continuous, then i) $A(t)=B(t)$ and $\lim _{n \rightarrow \infty} A A x_{n}=\lim _{n \rightarrow \infty} B B x_{n}=\lim _{n \rightarrow \infty} A B x_{n}=\lim _{n \rightarrow \infty} B A x_{n}$, where $\lim _{n \rightarrow \infty} A x_{n}=t$ and $\lim _{n \rightarrow \infty} B x_{n}=t$.

ii) If there exists $u \in X$ such that $A u=B u=t$, then $A B u=B A u$. In order to establish our result, we need a function $\emptyset: \mathbb{R}^{+} \rightarrow \mathbb{R}^{+}$satisfying $0<\emptyset(t)<t, t>0$.

\section{Main Results:}

Theorem 2.1: Let $(X, d)$ be a semi-metric space that satisfies (W4) and (H.E). Let $A, B, T$ and $S$ be self mappings of $X$, such that

i) $A X \subset T X$ and $B X \subset S X$

ii) $d(A x, B y) \leq \emptyset(\max \{\mathrm{d}(S x, \mathrm{Ty}), \mathrm{d}(S x, B y), d(T y, B y)\})$ for all $(x, y) \in X \times X$,

iii) The pair $(B, T)$ or $(A, S)$ satisfies E.A. property,

iv) The pair $(B, T)$ and $(A, S)$ are compatible mapping of type (E), and

v) $S X$ or $T X$ is a $d$-closed subset of $X$.

If one of the mapping $A, B, T$ and $S$ is continuous then $A, B, T$ and $S$ have a unique common fixed point.

\section{Proof:}

Since $(B, T)$ satisfies the property E.A. then there exists a sequence $\left\{{ }^{x}\right\}$ in $X$ such that $\lim _{n \rightarrow \infty} d\left(B x_{n}, t\right)=\lim _{n \rightarrow \infty} d\left(T x_{n}, t\right)=0$, for some $t \in X$.

Since $B X \subset S X$, there exists a sequence $\left\{y_{n}\right\}$ in $X$ such that $B x_{n}=S y_{n}$ and hence.

$\lim _{n \rightarrow \infty} d\left(S y_{n}, t\right)=0$ By the property H.E, we get $\lim _{n \rightarrow \infty} d\left(B x_{n}, T x_{n}\right)=\lim _{n \rightarrow \infty} d\left(S y_{n}, T x_{n}\right)=0$

If $S X$ is a $d$-closed subset of $X$ there exists a point $u \in X$ uch that $S u=t$

Also, we have

$$
\lim _{n \rightarrow \infty} d\left(B x_{n}, S u\right)=\lim _{n \rightarrow \infty} d\left(T x_{n}, S u\right)=\lim _{n \rightarrow \infty} d\left(S y_{n}, S u\right)=0
$$

Now, using condition (ii), we get 
$d\left(A u, B^{\left.x_{n}\right)} \leq \emptyset\left(\max \left\{\mathrm{d}\left(S u, \mathrm{~T} x_{n}\right), \mathrm{d}\left(S u, B x_{n}\right), d\left(T x_{n}, B x_{n}\right)\right\}\right)\right.$

Letting $n \rightarrow \infty$, we have

$\lim _{n \rightarrow \infty} d\left(A u, B^{x_{n}}\right)=0$. By the property W4, $\lim _{n \rightarrow \infty} d\left(T x_{n}, A u\right)=0$. This implies $A u=S u$.

Since $A X \subset T X$, there exists a point $w \in X$ such that, $A u=T w$. This implies $A u=S u=T w$

We claim that, $B w=T w$. Using condition (ii), we get

$d(A u, B w) \leq \emptyset(\max \{d(S u, T w), d(S u, B w), d(T w, B w)\})$

$$
\begin{aligned}
& \leq \emptyset(\max \{d(S u, T w), d(S u, B w), d(T w, B w)\}) \\
& \leq \emptyset(\max \{0, d(T w, B w), d(T w, B w)\}), \text { that is }
\end{aligned}
$$

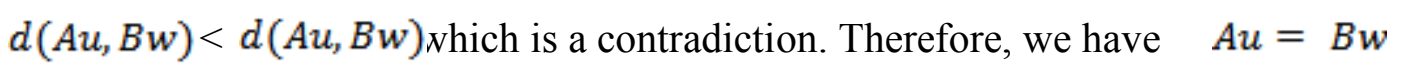

Hence, we have $A u=S u_{=} T w=B w$. For the existence of a common fixed point for four mappings of a semi-metric space we apply compatible mapping of type (E).

Suppose $S$ is continuous and $(A, S)$ is compatible mappings of type $(\mathrm{E})$, then by the proposition 1.1, we have $A u=S u$, then $A S u=S A u$.

Now, we have $A A u=A S u=S A u=S S u$.

We claim that $A u$ is a common fixed point of $A$ and $S$, if $A A u \neq A u$.

Using condition (ii), we get

$d(A u, A A u)=d(A A u, B w)$

$$
\begin{aligned}
& \leq \emptyset(\max \{d(S A u, T w), d(S A u, B w), d(T w, B w)\}) \\
& \leq \emptyset(\max \{d(A A u, A u), d(A A u, A u), 0\}), \text { that is }
\end{aligned}
$$

$<d(A u, A A u) d(A u, A A u)$, which is a contradiction.

Therefore, we have $A A u=A u$ which implies $A A u=S A u=A u$. Hence $A u$ is common fixed point of $A$ and $S$. By the proposition 1.1, we have $B T w=T B w$. Thus, we have $T T w=T B w=B T w=B B w$.

Similarly, we can show that $B w$ is common fixed point of $B$ and $T$. Since $A u=B w$,

$A u$ is a common fixed point of $A, B, T$ and $S$. If $\mathrm{z}$ is another common fixed point of $A, B, T$ and $S$,

$z \neq A u$ such that $A z=B z=T z=S z=z$.

Using condition (ii), we get

$$
\begin{aligned}
& d(A u, z)=d(A A u, B z) \leq \emptyset(\max \{d(S A u, T z), d(S A u, B z), d(T A u, B z)\}) \\
& \leq \emptyset(\max \{d(A u, z), d(A u, z), d(A u, z)\}) \\
& d(A u, z)<d(A u, z), \text { which is a contradiction. }
\end{aligned}
$$

So, we get $A u=z$. Hence $A, B, T$ and $S$ have a unique common fixed point.

Example 2.1. Let $X=(0,5]$ with semi-metric space $(X, d)$, define by $d(x, y)=(x-y)^{2}$. Define self-mappings $A, B, T$ and $S$ as

$A x=1$ for all $x$.

$B x=1$ if $x \leq 2$ and $x=3, B x=1+x$ if $2<x<3$

$S x=x$ if $x<4, \quad S x=4$ if $x>4$

,Tx $=1$ if $x \leq 2 T x=x-1$ if $2<x \leq 5$. 
Then $A, B, T$ and $S$ satisfy all the conditions of the above theorem and have a unique common fixed point at $x=1$.

In theorem 2.1, if we take $A=B$ and $T=S$ we have the following corollary.

Corollary 2.1: Let $(X, d)$ be a semi-metric space that satisfies (W4) and (H.E) and Let $A$ and $T$ be self mappings of $X$, such that

i) $A X \subset T X$.

ii) $d(A x, A y) \leq \emptyset(\max \{\mathrm{d}(T x, T y), \mathrm{d}(T x, A y), d(T y, A y)\})$ for all $(x, y) \in X \times X$

iii) The pair $(A, T)$ satisfies E.A. property.

iv) The pair $(A, T))$ are compatible of type (E)

v) $T X$ is a $d$-closed subset of $X$.

If one of the mapping $A$ and $T$ is continuous then $A$ and $T$ have a unique common fixed point.

Remarks : Our result generalizes the result of M. Aamri and D.El. Moutawakil [1], and S. H. Cho, G .Y .Lee, and J. S. Bae [4] extends the results of [7], [8] and other similar results in semi-metric space .

\section{References:}

[1] M. Aamri and D. El. Moutawakil, Common fixed points under contractive conditions in symmetric space, Appl. Math. E-Notes, 3(2003), 156-162.

[2] I. D. Arandelovic, D. S. Petkovic, A note on some fixed point results, Appl.Math. E- Notes, 9(2009), 254-261.

[3] S. H. Cho, G .Y. Lee, and J. S. Bae, On concidence and fixed point theorems in symmetric space, Hindawi publishing corporation 10(2008), 9 pages.

[4] M. Cicchese, Questioni di comptetezza e contrazioni in spazi metrici generalization Boll.Un.Mat.Italy.,13-A 5(1976), 175-179.

[5] M. Fr'echet, Sur quelques points du calcul fonctionnel, Rend. Circ. Mat. Palermo 22 (1906) $1-74$.

[6] T. L. Hicks, B. E. Rhoades, Fixed point theory in symmetric space with applications to probabilistic space, Nonlinear Analysis, 36(1999), 331-334.

[7] M. Imdad and Q. H. Khan, Six mappings satisfying a rational inequality, Radovi Matematicki, 9(1999), 251-260.

[8] M. Imdad, J. Ali, and L. Khan, Coincidence and fixed points in semi-metric spaces under strict conditions, J. Math. Anal. Appl.320(2006), 352-360.

[9] M. Imdad and J. Ali, Common fixed point theorems in semi-metric space employing a new implicit function and common property (E.A.), Bull. Belgium Soc. Simon Stevin, 16(2009), 421-433.

[10] K. Jha, R. P. Pant and K. B. Manandhar, A common fixed point theorem for compatible mappings of type (E) in metric space, Internat. J. Application and Applied Mathematics. (To Appear)

[11] G. Jungck, Compatible mappings and common fixed point, Internat. J. Math. Sci., 9(1986),771-779.

[12] K. Menger, Untersuchungen uber allgemeine, Math Annalen, 100(1928),75-163.

[13] R. P. Pant, Common fixed point of non-commuting mappings, J. Math. Anal. Appl. 188(1994), 436-440.

[14] M. R. Singh and M. Y. Singh Compatible mappings of type (E) and common fixed point theorems of Meir-Keeler type, Internat. J. Math. Sci. \& Engg. Appl.1 2 (2007), 299-315.

[15] W. A. Wilson,On semi-metric space, American .J. Math, 53(1931),361-373. 